\title{
Glycemic Variability and Mean Weekly Glucose in the Evaluation and Treatment of Blood Glucose in Gestational Diabetes Mellitus; Evidence for Lower Neonatal Complications
}

Tatiana Munhoz da Rocha Lemos Costa ${ }^{1 *}$, Josiane Melchioretto Detsch', Augusto Pimazoni-Netto ${ }^{2}$, Ana Cristina Ravazzani de Almeida1, Shoshana Sztal-Mazer ${ }^{1}$, Larissa Marques Tondin de Oliveira ${ }^{3}$, Dênis José Nascimento ${ }^{4}$ and Rosângela Roginski Réa ${ }^{1}$

Service of Endrocrinology and Metabolism of the Federal University of Paraná, Brasil

Service of Diabetes and Hypertension Control of the Federal University of São Paulo, Brasil

3Service of Nutrition of the Federal University of Paraná, Brasil

${ }^{4}$ Service of High Risk Pregnancy of the Federal University of Paraná, Brasil

\begin{abstract}
Background: Gestational Diabetes Mellitus (GDM) incurs a risk of morbidity and mortality to mother, fetus and subsequent neonate which necessitates intensive monitoring and treatment. Recently, weekly blood glucose and, more importantly, glycemic variability have been explored as means of assessing glycemic control and altering management in type 2 diabetes. For the first time in GDM, we aimed to assess the applicability of these methods and determine whether they affect complications.
\end{abstract}

Methods: We conducted a prospective, randomized un-blinded single centre study $(n=30)$ where the intervention arm $(n=15)$ was assessed and managed using seven point self blood glucose monitoring, glycemic variability and mean blood glucose while the control arm received standard GDM management. Blood glucose variables, glycemic target achievement and outcome variables to mother, fetus and neonate were recorded.

Results: Weight and $\mathrm{HbA} 1 \mathrm{c}$ did not differ significantly between or within groups over the study period and $\geq 80 \%$ of participants in both groups achieved glycemic targets $(p=0.850)$. In the intervention arm, glycemic variability decreased significantly $(p=0.016)$, however the weekly mean blood glucose did not. The difference between groups in therms of gestational hypertension and pre-eclampsia did not reach statistical significance. Fetal abnormalities only occurred in the control group $(n=4,26.66 \%, p=0.032)$. Furthermore, there were more neonatal complications in the control group compared to the intervention group $(n=8 ; 53.33 \%$ versus $n=1 ; 6.66 \% ; p=0.007)$

Conclusions: We have shown that glycemic variability and mean weekly glucose measurements can easily be implemented in the management of GDM. As both groups achieved target glycemic control and glycemic variability was the only parameter that improved over the study period, we believe that this may explain improved fetal and neonatal outcomes in the intervention arm. Larger, properly powered studies are required to further explore this finding.

Keywords: Glycemic variability; Mean weekly glucose; Gestational diabetes mellitus; Neonatal complications; Fetal complications

\section{Introduction}

Gestational diabetes mellitus (GDM) is defined as glucose intolerance of varying degrees, which appears, or is first diagnosed, during pregnancy and may or may not persist after delivery $[1,2]$. It occurs in approximately $7 \%$ of pregnancies [3] and incurs a risk of morbidity and mortality to mother, fetus and subsequent neonate $[4,5]$. Therefore, screening for early diagnosis, followed by intensive treatment is necessary to avoid such potential complications [6,7]. The methods for assessment of glycemic control are divided into traditional methods (tests for blood glucose, fructosamine and glycosylated hemoglobin $(\mathrm{HbA} 1 \mathrm{c})$ ), latest methods (continuous monitoring of interstitial glucose (CGMS)) and new proposed methods (seven-point self-monitored blood glucose (SMBG) profiles along with calculation of weekly mean blood glucose (WMG) and glycemic variability (GV)).

Although the concept of glycemic variability has been described in the literature for many year [8-13], it was brought to the fore in 2008 when Monnier, Colette and Owen wrote a review about its importance and potential applicability to everyday diabetes management, should the right tools for its measurement be developed. Glycemic variability refers to glycemic swings, peaks and nadirs, around an average. It has been observed that highly variable blood glucose levels may be more deleterious to endothelial function and lead to greater oxidative stress, hence diabetes complications, than sustained hyperglycemia [14,15] While $\mathrm{HbA1C}$ provides a good measure of average blood glucose in the medium term (approximately 12 weeks); it does not accurately reflect glycemic variability, nor does it reflect short term glycemic control [16]. Thus, a number of methods have been proposed to assess these latter two variables and treatment adequacy. To be applicable to clinical practice, they should be reliable, inexpensive, easy to implement and user-friendly. At the 70th Congress of the American Diabetes Association, Pimazoni-Nettos's group reported improved glycemic control in Type 2 Diabetes (T2D) when they used seven point SMBG 3 days per week to calculate GV and WMG along with weekly clinic visits

*Corresponding author: Tatiana Munhoz da Rocha Lemos Costa, Service of Endrocrinology and Metabolism of the Federal University of Paraná, Brasil, E-mail: tatimrlemos@yahoo.com.br

Received July 20, 2011; Accepted September 02, 2011; Published Septembe 06, 2011

Citation: Lemos Costa TMR, Detsch JM, Pimazoni-Netto A de Almeida ACR, Sztal-Mazer S, et al. (2011) Glycemic Variability and Mean Weekly Glucose in the Evaluation and Treatment of Blood Glucose in Gestational Diabetes Mellitus; Evidence for Lower Neonatal Complications. J Diabetes Metab 2:137. doi:10.4172/2155-6156.1000137

Copyright: (C) 2011 Lemos Costa TMR, et al. This is an open-access article distributed under the terms of the Creative Commons Attribution License, which permits unrestricted use, distribution, and reproduction in any medium, provided the original author and source are credited. 
Citation: Lemos Costa TMR, Detsch JM, Pimazoni-Netto A, de Almeida ACR, Sztal-Mazer S, et al. (2011) Glycemic Variability and Mean Weekly Glucose in the Evaluation and Treatment of Blood Glucose in Gestational Diabetes Mellitus; Evidence for Lower Neonatal Complications. J Diabetes Metab 2:137. doi:10.4172/2155-6156.1000137

[17]. Clearly, a means of measuring short term glycemic fluctuations, and the facility to adjust treatment based on the results of these tests, is desirable in GDM where the disease itself is often short term and its potential complications result from this short term hyperglycemia, or perhaps dysglycemia. Therefore, in our study, we implemented these methods to assess and manage glycemic control for the first time in a GDM population.

Our primary objective was to demonstrate that WMG and GV can be used to assess near real time blood glucose and treatment in patients with GDM, while allowing weekly therapy adjustments to improve glycemic control in the short term; that they are practical, easy to implement and user-friendly methods which are independent of HbAlc. As a secondary objective, we aimed to determine whether better glycemic control with the use of WMG and GV reduce the number of maternal, fetal and neonatal complications attributed to GDM.

\section{Material and Methods}

We conducted a prospective, randomized un-blinded single centre study between 01/02/2010 and 15/11/2010 in the Diabetes Mellitus and Pregnancy outpatient clinic at the Hospital de Clínicas da Universidade Federal do Paraná (HC / UFPR). The study was approved by the hospital's Ethics Committee on Human Research. All patients signed an informed consent form before enrollment. Inclusion criteria consisted of GDM, diagnosed by an Oral Glucose Tolerance Test (OGTT) with 75 grams of glucose, according to guidelines set by the Brazilian Ministry of Health; and attendance at the GDM clinic which is located within the high risk pregnancy clinic, to which patients were referred when diagnosed with GDM [18]. Exclusion criteria were preexisting type 1 or 2 diabetes mellitus, refusal to use insulin, refusal to self monitor, inability to attend appointments and inadequate level of understanding.

Patients were randomized, using the lottery principle, in to 2 groups: the intervention arm and the control arm. All patients were provided with individual dietary counseling by a nutritionist upon entry into the study. The prescribed diet was equal for all and specific to pregnant women with diabetes, with adequate amounts of calories, macro and micronutrients, and based on carbohydrate counting. All patients had HbAlc monitored monthly, while ultrasound follow-up was arranged by the obstetric service as they saw fit.
Patients in the intervention arm underwent more intensive monitoring and follow-up than that provided with our standard care. On Mondays, Tuesdays and Wednesdays, seven point SMBG were recorded using the ACCU-CHEK ${ }^{\circledR}$ Active monitor preprandially, 2 hours post prandially and at $3 \mathrm{am}$. Then, each week, on Thursday, patients were reviewed by the GDM endocrine team. Data from their monitors were downloaded; and, using the ACCU-CHEK ${ }^{\circledR} 360^{\circ}$ software, WMG, GV and the general times at which hyperglycemia occurred were calculated. The goals of glycemic control in this group were a WMG $\leq 100 \mathrm{mg} / \mathrm{dl}$ and a $\mathrm{GV} \leq 30 \mathrm{mg} / \mathrm{dl}$. Insulin was commenced if these targets were not reached within one week.

Patients in the control arm received standard care which involved GDM endocrine team review monthly until 24 weeks' gestation, fortnightly until 32 weeks and weekly thereafter. Glycemic control was assessed using a glycemic profile done prior to the consultation whereby venous blood glucose was measured at the hospital biochemical laboratory at 8:00 am, 10:00 am and 2:00 pm corresponding to fasting, 2 hours post-breakfast and 2 hours post-lunch readings. As our standard care does not involve providing glucose monitors, neither GV nor WMG were measured in this group. The goals of glycemic control in this group were a fasting glucose $\leq 95 \mathrm{mg} / \mathrm{dl}$ and a 2 hours postprandial glucose $\leq 120 \mathrm{mg} / \mathrm{dl}$. Insulin was initiated if these targets were not reached within two weeks.

The maternal baseline data collected were age, gestational age at diagnosis, gestational age when monitoring began, fasting blood glucose on screening, results of OGTT, pre-pregnancy body mass index (BMI) and BMI at diagnosis. During our follow-up, weight gain during pregnancy, treatment (diet or diet plus insulin), dose and type of insulin, number of insulin injections per day, values of capillary or venous blood glucose (depending on the group to which the patient was randomized) and $\mathrm{HbAlc}$ levels were recorded. Complications seen on ultrasound, gestational hypertension, preeclampsia, intrauterine death, gestational age at delivery, mode of delivery and perinatal death were all documented. Neonatal outcome data recorded were intensive care admission, size for gestational age (large, LGA or small, SGA), hyperbilirubinemia (requiring phototherapy), shoulder dystocia, hypoglycemia defined as a blood glucose $<40 \mathrm{mg} / \mathrm{dl}$ and respiratory distress.

Insulin, when needed, was provided by the Brazilian public health

\begin{tabular}{|c|c|c|c|}
\hline Baseline Characteristics & Intervention Arm $(n=15)$ & Control Arm (n=15) & $P$ value \\
\hline Age (Years) & $34,87 \pm 4,45$ & $32,53 \pm 4,94$ & 0,185 \\
\hline Gestational Age when monitoring began(Weeks) & $31,53 \pm 2,85$ & $28,93 \pm 2,54$ & 0,013 \\
\hline Gestational Age at GDM Diagnosis (Weeks) & $25,86 \pm 3,52$ & $25,80 \pm 2,33$ & 0,136 \\
\hline Fasting Blood Glucose on Screening (mg / dl) & $91,86 \pm 12,09$ & $88,06 \pm 16,23$ & 0,283 \\
\hline Fasting Blood Glucose on OGTT (mg / dl) & $98,5 \pm 13,59$ & $98,3 \pm 16,96$ & 0,416 \\
\hline Postprandial Blood Glucose after 2 h on OGTT (mg / dl) & $171 \pm 22,38$ & $166 \pm 18,16$ & 0,443 \\
\hline Pre-pregnancy BMI (kg / m²) & $27,83 \pm 3,44$ & $29,44 \pm 7,04$ & 0,436 \\
\hline BMI at Diagosis of DMG $\left(\mathrm{kg} / \mathrm{m}^{2}\right)$ & $31,12 \pm 4,61$ & $32,82 \pm 6,26$ & 0,263 \\
\hline Glycosylated hemoglobin (\%) & $5,8 \pm 0,44$ & $5,6 \pm 0,46$ & 0,850 \\
\hline
\end{tabular}

Legend: Baseline patients characteristics

Table 1: Baseline Maternal Characteristics.

\begin{tabular}{|l|l|l|l|l|}
\hline Change in weight & Intervention Arm & \multicolumn{3}{l|}{ Control Arm } \\
\hline Weight Gain & 9 patients & $3,95 \pm 2,91 \mathrm{~kg}$ & 11 patients & $2,98 \pm 1,72 \mathrm{~kg}$ \\
\hline Weight Loss & 5 patients. & $1,8 \pm 0,95 \mathrm{~kg}$ & 3 patients & $3,2 \pm 0,6 \mathrm{~kg}$ \\
\hline
\end{tabular}

Legend: Comparison between the two groups in relation to weight gain throughout the study

Table 2: Change in Weight Over Study Period 
Citation: Lemos Costa TMR, Detsch JM, Pimazoni-Netto A, de Almeida ACR, Sztal-Mazer S, et al. (2011) Glycemic Variability and Mean Weekly Glucose in the Evaluation and Treatment of Blood Glucose in Gestational Diabetes Mellitus; Evidence for Lower Neonatal Complications. J Diabetes Metab 2:137. doi:10.4172/2155-6156.1000137

Page 3 of 5

service. Blood was taken and analyzed at the HC/UFPR pathology laboratory. Ultrasounds were performed by the hospital's obstetric service. Glucose meters, test strips and software used were donated by Roche Diagnostics Brazil.

Data were analyzed using the Statistical Package for Social Sciences (SPSS) version 17 (SPSS Inc., Chicago, IL, USA).Initially, all variables were analyzed descriptively. Continuous variables were summarized as the mean \pm SD. For qualitative variables, we calculated absolute and relative frequencies. To exclude the null hypothesis when analyzing the means of two groups, we used the Student $t$ test. When the data was not normally distributed, we used the nonparametric Mann-Whitney test. To compare proportions between groups, we used the chi-square test or Fisher's exact test. A P values $\leq 0.05$ was considered significant.

\section{Results}

During the study period, 30 patients were randomly assigned either to the intervention group (15 patients) or to the control group (15 patients). Baseline characteristics did not significantly differ between these groups (Table 1) with the exception of when glucose monitoring began. Patients in the intervention arm began an average of 2.6 weeks later than those in the control arm. Adherence to the protocol was $100 \%$ for both groups. This involved attendance at consultations, prescribed therapy, blood glucose tests and laboratory investigations requested.

Ten patients in the intervention group (66.66\%) versus 6 patients in the control group (40\%) required insulin, but this difference was not statistically significant $(\mathrm{p}=0.272)$. Regarding the type of insulin used, the only significant difference was that five patients in the intervention arm used regular insulin in isolation while none of the controls were managed in this way $(\mathrm{p}=0.021)$. Three patients both in the intervention

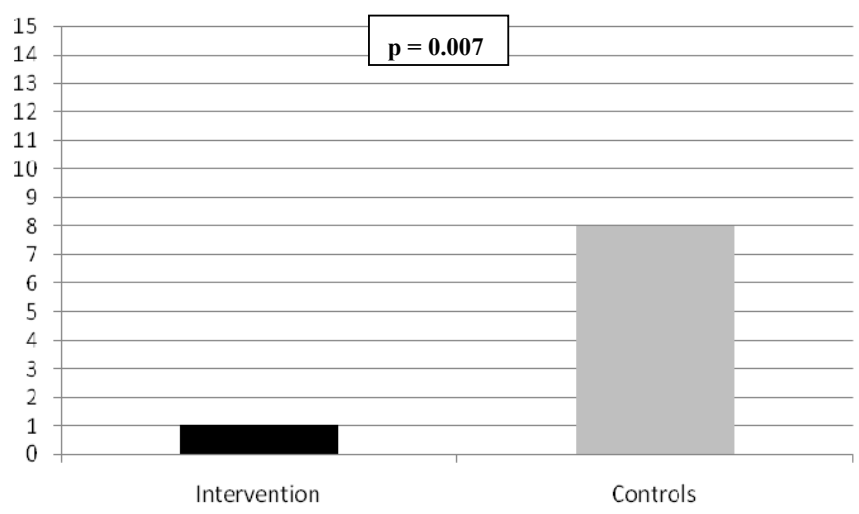

Legend: Number of neonatal complications in each group

Figure 1: Number of neonates in whom any complication developed.

\begin{tabular}{|l|l|l|l|l|l|}
\hline Complications & \multicolumn{2}{|c|}{ Intervention Arm } & \multicolumn{2}{|c|}{ Control Arm } & P value \\
\hline Hyperbilirubinemia & 1 & $6,66 \%$ & 3 & $20 \%$ & 0,299 \\
\hline ICU admission & 1 & $6,66 \%$ & 5 & $33,33 \%$ & 0,084 \\
\hline Hypoglycemia & 0 & $0 \%$ & 3 & $20 \%$ & 0,122 \\
\hline LGA & 0 & $0 \%$ & 5 & $33,33 \%$ & 0,021 \\
\hline SGA & 0 & $0 \%$ & 2 & $13,33 \%$ & 0,241 \\
\hline Respiratory Distress & 0 & $0 \%$ & 2 & $13,33 \%$ & 0,241 \\
\hline Shoulder Dystocia & 0 & $0 \%$ & 0 & $0 \%$ & Not applicable \\
\hline
\end{tabular}

Legend: Neonatal complications in each group

Table 3: Neonatal Complications. and control group used only NPH, while two in the intervention and three in control group used a combination of NPH and Regular insulin. There was no statistically significant difference in the total insulin dose or the number of injections per day between groups. Specifically, 12.6 \pm 13.16 versus $16.66 \pm 13.36$ units $(\mathrm{p}=0.900)$ were given in $2.10 \pm 0.99$ versus $1.83 \pm 0.75(\mathrm{p}=0.550)$ injections each day in the intervention and control arms respectively.

The change in weight between GDM diagnosis and delivery was not statistically different between groups $(\mathrm{p}=0.852)$ (Table 2$)$. Baseline $\mathrm{HbA1C}$ did not differ between groups, being $5.8 \pm 0.44 \%$ in the intervention arm versus $5.63 \pm 0.53 \%$ in the control arm $(p=0.400)$ ). Final HbA1c did not differ between $(\mathrm{p}=0.610)$ or within groups either, whereby the intervention group showed a decrease of $0.2 \%(\mathrm{p}=0.870)$ while the control group showed an increase of $0.18 \%(\mathrm{p}=0.850)$.

As stated previously, WMG and GV were only evaluated in the intervention arm. At the start of monitoring the average WMG was $103.05 \pm 18.54 \mathrm{mg} / \mathrm{dl}$ and at the end of the study it was $97.09 \pm 6.55$ $\mathrm{mg} / \mathrm{dl}$, however this reduction was not statistically significant $(\mathrm{p}=$ $0.125)$. The GV, however decreased significantly from $21.53 \pm 10.84$ $\mathrm{mg} / \mathrm{dl}$ to $17.99 \pm 5.43 \mathrm{mg} / \mathrm{dl}(\mathrm{p}=0.016)$ over the study period. There was no statistically significant difference between groups in terms of reaching target glycemic control $(p=0.850)$, though it must be noted that these tragets were inherently group specific. At the end of the study 12 of 15 patients $(80 \%)$ in the intervention group were within target (MWG and GV). Two had only GV within target while one patient did not achieve target values for either parameter. In the control group, 13 patients achieved target blood glucose levels fasting and 2 hours postprandial levels. There was a tendency towards earlier delivery in the control group $(37.53 \pm 1.80$ weeks versus $38.33 \pm 1.75$ weeks for the intervention group $(\mathrm{p}=0.058)$ ). Cesarean delivery was performed in 14 patients in intervention group $(93.33 \%)$ and 11 patients in control group $(73.33 \%)$, with no statistical difference between groups $(\mathrm{p}=$ $0.165)$.

Regarding maternal complications, gestational hypertension occurred in three patients in the intervention group (20\%) and in 7 patients in the control group (46.66\%), however no statistically significant difference between groups was found $(\mathrm{p}=0.123)$. Preeclampsia was diagnosed in one patient in the intervention group $(6.66 \%)$ and in 4 patients in the control group (26.66\%), but again, this difference was not statistically significant $(\mathrm{p}=0.165)$. As fetal abnormalities visualized on ultrasound were only found in the control group (4 patients $-26.66 \%$ of patients) there was a statistically significant difference between groups $(p=0.032)$. The changes seen were: oligohydramnios [2], intrauterine growth retardation [1], and an increase in fetal abdominal circumference [1]. These fetuses, along with 4 other controls, went on to develop neonatal complications. That is, 8 neonates in the control arm $(53.33 \%)$ versus only lin the intervention arm $(6.66 \%)$ suffered complications, a clearly significant difference in terms of neonatal complications $(\mathrm{p}=0.007)$ (Figure 1$)$. When evaluated separately, the only complication which reached statistical significance was LGA $(\mathrm{p}=0.021)$ (Table 3$)$. There was no perinatal death or shoulder dystocia in either group.

\section{Discussion}

The Education and Control of Diabetes Group from The Kidney and Hypertension Hospital, Universidade Federal de São Paulo, used seven point SMBG along with GV and WMG calculation to assess glycemic control in patients with T2D [17]. In our study, we used the same methods to evaluate and manage patients with GDM. The 

Glucose in the Evaluation and Treatment of Blood Glucose in Gestational Diabetes Mellitus; Evidence for Lower Neonatal Complications. J Diabetes Metab 2:137. doi:10.4172/2155-6156.1000137

time between GDM diagnosis and the commencement of treatment was longer than ideal in both groups. Unfortunately, this is a function of the strained Brazilian public health system: patients needed to be identified and then needed to wait for an appointment to be seen at our outpatient clinic. By chance, glucose monitoring began earlier in the control arm. This observation may have favored the controls in terms of a positive outcome, given that GDM is a disease which demands urgent treatment. The absolute adherence shown by all patients probably reflects the exceptional dedication women, and even their families, have to their own health when they are pregnant.

Although there was no statistical difference in the number of patients requiring insulin between groups, more patients in the intervention arm used regular insulin alone. This was due to the insight into the timing of peak postprandial blood glucose levels aided by the ACCU-CHEK ${ }^{\circledR} 360^{\circ}$ software, as well as the fact that only patients in the intervention group monitored blood glucose levels after dinner. These observations reinforce the definition of GDM as a condition of glucose intolerance whose diagnosis requires the OGTT. The number of insulin injections per day and the total dose used during pregnancy were not statistically different between groups. Given patients in both groups met their respective glycemic targets equally, this attests to similar patient characteristics; which translate to similar insulin requirements across groups.

The $\mathrm{HbAlc}$ test is considered a medium term means of assessing glycemic control, the applicability of which is inherently limited in GDM, given its short treatment time and need for rapid responses to small increments in blood glucose. It reflects glucose levels retrospectively and not in "real time". The various available laboratory methods and hence loose definition of the normal range, in addition to the large number of clinical conditions which can result in false positives and negatives are all potential methodological difficulties which can occur. In turn, the sensitivity of this test is low and well defined criteria in GDM are lacking. Indeed, we found no difference in initial and final HbAlc levels between or within groups. Thus, we advocate that $\mathrm{HbAlc}$ should not be used as a determining factor to start insulin therapy in GDM.

While the glycemic variability decreased between the beginning and the end of the study, the WMG did not show a statistically significant decline. This could be attributed to the fact that patients were given dietary advice at the initial consultation, prior to the first 7 points profile. A delay in dietary change in a condition with limited treatment time would have been unacceptable from an ethical standpoint. Indeed, diet may be the only treatment necessary for a situation characterized by "impaired glucose tolerance or low pancreatic reserve" such as that seen in DMG [19-21]. Furthermore, GDM causes a relatively mild elevation in blood glucose, so a significant change in WBG is difficult to demonstrate. At the end of their pregnancies, $86.6 \%$ of patients in the control group and $80 \%$ of patients in the intervention group had readings which were within their respective glycemic control objectives hence no statistically significant difference between groups was found. This indicates that there was no difference in the number of patients in whom diabetes was controlled and that our current standard practice with GDM is adequate in terms of glycemic control.

Maternal complications associated with uncontrolled gestational diabetes were not significantly different between groups. However complications seen on ultrasound were more frequent in the control group. The perinatal complications of GDM and glucose intolerance are well known in the literature [22-26]. Consitent with this, we found a significant difference between groups in terms of the number of neonates who suffered complications: 8 patients in the control group versus in intervention group. Interestingly, however, both groups were within target glycemic control which begs the question: why did only one group suffer the complications usually seen in GDM? We can deduce that the severity of GDM was similar between groups based on fasting glucose, OGTT results, initial BMI and amount of insulin used. Compliance rates and number of patients within target glycemic control attest to the fact that adequate average glycemic control was achieved in both groups. As our primary objective was to test the use of GV as a parameter with which to assess and adjust management in GDM, this value wasn't actually measured in the control group. Nevertheless, it was the only statistically significant point of difference over the study period. Therefore, we can infer that it was the possibly lower glycemic variability in the intervention group that may have at least contributed to the lower rate of perinatal complications seen.

In general, many of our results, such as maternal complications, were not found to be statistically significant perhaps because our sample size was too small to have the power to demonstrate this. For this variable, wheter the proportion mantained, the number of patients necessary to achieve a statistical significance would be twenty (calculated by the power sample size). Nevertheless, the results above that did reach significance, should alert us to the importance of these findings.

\section{Conclusion}

We have shown, in patients with GDM, that the seven point SMBG 3 days per week to calculate GV and WMG along with weekly clinic visits, was practical, easy to implement, associated with excellent compliance rates, enabled better visualization of blood glucose levels and facilitated therapy adjustments. We have demonstrated that this new method was associated with a lower rate of fetal and neonatal complications compared with standard treatment and follow-up, despite the relatively small number of patients and the acheivement of glycemic targets in both groups. This novel finding suggests that greater glucose variability may cause deleterious effects to the fetus and hence neonate. This study is the first of its kind to use a new method of assessing glycemic control in patients with GDM and, after necessary regimen adjustments, to demonstrate a change in outcome likely based on GV. Our favorable results justify additional research with large, properly powered trials.

\section{References}

1. American Diabetes Association (2006) Diagnosis and classification of diabetes mellitus. Diabetes Care 29: 43-48.

2. Vilar L. Endocrinologia Clínica (2006) $3^{a}$ ed. Rio de Janeiro: Guanabara Koogan p. 630-642.

3. American Diabetes Association (2004) Gestational Diabetes Mellitus. Diabetes Care 27: 88-90.

4. Buchanan TA, Xiang A, Kjos SL, Watanabe R (2007) What is gestational diabetes? Diabetes Care 30: 105 -111.

5. Buchanan TA, Xiang AH (2005) Gestational diabetes mellitus. J Clin Investig 115: 485-491.

6. Sociedade Brasileira de Endocrinologia e Metabologia (2006) Projeto Diretrizes: Diabetes Mellitus Gestacional 1-12.

7. Hillier TA, Vesco KK, Pedula KL, Beil TL, Whitlock EP, et al. (2008) Screening for gestational diabetes mellitus: A systematic review for the U.S. Preventive Services Task Force. Ann Intern Med 148: 766-775.

8. Service FJ, Molnar GD, Rosevear JW, Ackerman E, Gatewood LC, et al. (1970) Mean amplitude of glycemic excursions, a measure of diabetic instability. Diabetes 19: 644-655. 
Citation: Lemos Costa TMR, Detsch JM, Pimazoni-Netto A, de Almeida ACR, Sztal-Mazer S, et al. (2011) Glycemic Variability and Mean Weekly Glucose in the Evaluation and Treatment of Blood Glucose in Gestational Diabetes Mellitus; Evidence for Lower Neonatal Complications. J Diabetes Metab 2:137. doi:10.4172/2155-6156.1000137

Page 5 of 5

9. Kilpatrick ES, Rigby AS, Atkin SL (2006) The effect of glucose variability on the risk of microvascular complications in type 1 diabetes. Diabetes Care 29 1486-1490.

10. Wentholt IM, Kulik W, Michels RP, Hoekstra JB, DeVries JH (2008) Glucose fluctuations and activation of oxidative stress in patients with type 1 diabetes. Diabetologia 51: 183-190.

11. Kovatchev BP, Otto E, Cox D, Gonder-Frederick L, Clarke W (2006) Evaluation of a new measure of blood glucose variability in diabetes. Diabetes Care 29 2433-2438.

12. Monnier L, Colette C, Owens DR (2008) Type 2 diabetes: a well-characterised but suboptimally controlled disease Can we bridge the divide? Diabetes Metab 34: 207-216.

13. Colette C, Monnier L (2007) Acute glucose fluctuations and chronic sustained hyperglycemia as risk factors for cardiovascular diseases in patients with type 2 diabetes. Horm Metab Res 39: 683-686.

14. Monnier L, Colette C (2008) Glycemic Variability - Should We And Can We Prevent It? Diabetes Care 31: 150-154

15. Ceriello A, Esposito K, Piconi L, Innat MA, Thorpe JE, et al. (2008) Oscillating Glucose Is More Deleterious to Endothelial Function and Oxidative Stress Than Mean Glucose in Normal and Type 2 Diabetic Patients. Diabetes 57: 13491354.

16. Gorus F, Mathieu C, Gerlo E (2006) How should HbA1c measurements be reported? Diabetologia 49: 7-10.

17. Pimazoni-Netto A, Zanella MT, Rodbard R, Pires (2010) Rapid improvement in Glycemic Control, Variability and A1c within 6 weeks using 7 point glycemic profiles 3 days per week and weekly clinic visit: a randomized controlled trial in Type 2 diabetes. Diabetes 59: A134 (Abstract 497-P).
18. Miranda Pacs, Reis R (2008) Diabetes Mellitus Gestacional. Arquivos Brasileiros de Endocrinologia 54: 447-480.

19. Yang X, Hsu-Hage B, Zhang H, Zhang C, Zhang Y, et al. (2002) Women with impaired glucose tolerance during pregnancy have significantly poor pregnancy outcomes. Diabetes Care 25: 1619-1624.

20. Rudge MV, Calderon IM, Ramos MD, Abbade JF, Rugolo LM (2000) Perinata outcome of pregnancies complicated by diabetes and by maternal daily hyperglycemia not related to diabetes. A retrospective 10-year analysis. Gynecol Obstet Invest 50: 108-112.

21. Vidaeff AC, Yeomans ER, Ramin SM (2003) Gestational diabetes: a field of controversy. Obstet Gynecol Surv 58: 759-769.

22. Östlund, Hanson U, Björklund A, Hjertberg R, Eva N, et al. (2003) Materna and Fetal Outcomes if Gestational Impaired Glucose Tolerance Is Not Treated. Diabetes Care 26: 2107-2111.

23. Metzger BE, Buchanan TA, Coustan DR, de Leiva A, Dunger DB (2007) Summary and Recommendations of the Fifth International WorkshopConference on Gestational Diabetes Mellitus. Diabetes Care 30: 251-260.

24. Jones CW (2001) Gestational diabetes and its impact on the neonate. Neonata Netw 20: 17-23.

25. Catalano PM, Kirwan JP, Haugel-de Mouzon S, King J (2003) Gestational diabetes and insulin resistance: role in short- and longterm implications for mother and fetus. J Nutr. 133: 1674-1683.

26. Grissa O,Yessoufou A, Mrisak I, Hichami A, Amoussou-Guenou D, et al (2010) Growth factor concentrations and their placental mRNA expression are modulated in gestational diabetes mellitus: possible interactions with macrosomia. BMC Pregnancy Childbirth 10: 7. 